\title{
Empirical Bayes Inference for the Parameter of Power Distribution Based on Ranked Set Sampling
}

\author{
Naiyi Li, ${ }^{1,2}$ Yuan Li, ${ }^{1}$ Yongming $\mathrm{Li}^{3}$, and Yang $\mathrm{Liu}^{4}$ \\ ${ }^{1}$ School of Economics and Statistics, Guangzhou University, Guangzhou 510006, China \\ ${ }^{2}$ College of Science, Guangdong Ocean University, Zhanjiang 524088, China \\ ${ }^{3}$ Department of Mathematics, Shangrao Normal University, Shangrao 334001, China \\ ${ }^{4}$ College of Economics, Jinan University, Guangzhou 510632, China
}

Correspondence should be addressed to Naiyi Li; linaiyi1979@163.com

Received 13 January 2015; Revised 3 May 2015; Accepted 5 May 2015

Academic Editor: Chris Goodrich

Copyright (C) 2015 Naiyi Li et al. This is an open access article distributed under the Creative Commons Attribution License, which permits unrestricted use, distribution, and reproduction in any medium, provided the original work is properly cited.

This research is based on ranked set sampling. Through the analysis and proof, the empirical Bayes test rule and asymptotical property for the parameter of power distribution are obtained.

\section{Introduction}

Ranked set sampling (RSS) is now regarded as an effective tool in statistical inference and important alternative to simple random sampling. RSS was first applied in agriculture [1]. In recent years, it has been applied more and more in areas such as environment and ecology. It is also a potential for the method to be successfully applied in industrial statistics and sociology, for which readers can refer to the monograph by Chen et al. [2].

Empirical Bayes (EB) approach was originally proposed by Robbins [3, 4], soon after it had been studied in the literature [5-9]. Up to now, EB methods are commonly based on simple random sampling (SRS). As a statistical procedure based on the RSS would perform better than its counterpart based on simple random sampling (SRS), a natural idea is to develop EB methods based on RSS. We will construct empirical Bayes test rule for the parameter of power distribution based on RSS.

Let $X$ have a conditional density function for given $\theta$ :

$$
f(x \mid \theta)=(\theta x)^{-1} \exp \left\{\theta^{-1} \ln x\right\},
$$

where $\theta$ is an unknown parameter, $\Omega=\{x \mid x>1\}$ is sample space, and $\Theta=\{\theta \mid \theta>0\}$ is parameter space.

In the paper, the main motivation of studying the density of (1) is as follows: (a) it is widely used in the fields of reliability and economy and so on; (b) it is a common distribution in reliability distribution; (c) on the basis of studying it, we intend to further consider empirical Bayes inference for some other distributions based on ranked set sampling. In the paper, we study the following test problem:

$$
H_{0}: \theta \leq \theta_{0} \Longleftrightarrow H_{1}: \theta>\theta_{0},
$$

where $\theta_{0}$ is a given positive constant.

To construct test function, we take loss function:

$$
\begin{aligned}
& L_{0}\left(\theta, d_{0}\right)= \begin{cases}0, & \theta \leq \theta_{0} \\
a\left[1-\left(\frac{\theta_{0}}{\theta}\right)\right], & \theta>\theta_{0} ;\end{cases} \\
& L_{1}\left(\theta, d_{1}\right)= \begin{cases}a\left[\left(\frac{\theta_{0}}{\theta}\right)-1\right], & \theta \leq \theta_{0} \\
0, & \theta>\theta_{0},\end{cases}
\end{aligned}
$$

where $a>0, d=\left\{d_{0}, d_{1}\right\}$ is action space, respectively, and $d_{0}$ and $d_{1}$ imply acceptance and rejection of $H_{0}$.

Suppose that the prior distribution $G(\theta)$ of parameter $\theta$ is unknown, we can get randomized decision function:

$$
\delta(x)=P\left(\text { accept } H_{0} \mid X=x\right) .
$$


Then, the risk function of $\delta(x)$ is given by

$$
\begin{aligned}
& R(\delta(x), G(\theta))=\int_{\Theta} \int_{\Omega}\left[L_{0}\left(\theta, d_{0}\right) f(x \mid \theta) \delta(x)\right. \\
& \left.\quad+L_{1}\left(\theta, d_{1}\right) f(x \mid \theta)(1-\delta(x))\right] d x d G(\theta) \\
& \quad=a \int_{\Omega} \beta(x) \delta(x) d x+C_{G},
\end{aligned}
$$

where

$$
\begin{aligned}
C_{G} & =\int_{\Theta} L_{1}\left(\theta, d_{1}\right) d G(\theta), \\
\beta(x) & =\int_{\Theta}\left[1-\left(\frac{\theta_{0}}{\theta}\right)\right] f(x \mid \theta) d G(\theta) .
\end{aligned}
$$

The marginal density function of $X$ is shown by

$$
\begin{aligned}
f_{G}(x) & =\int_{\Theta} f(x \mid \theta) d G(\theta) \\
& =\int_{\Theta}(\theta x)^{-1} \exp \left\{\theta^{-1} \ln x\right\} d G(\theta) .
\end{aligned}
$$

Applying (6), we get

$$
\begin{aligned}
\beta(x)= & \int_{\Theta}\left(1-\frac{\theta_{0}}{\theta}\right) f(x \mid \theta) d G(\theta) \\
= & \int_{\Theta}\left(1-\theta_{0}+\theta_{0}-\frac{\theta_{0}}{\theta}\right) f(x \mid \theta) d G(\theta) \\
= & \int_{\Theta}\left(1-\theta_{0}\right) f(x \mid \theta) d G(\theta) \\
& +\int_{\Theta}\left(\theta_{0}-\frac{\theta_{0}}{\theta}\right) f(x \mid \theta) d G(\theta) \\
= & \left(1-\theta_{0}\right) f_{G}(x)-\theta_{0} x \\
& \cdot \int_{\Theta}\left(-1+\frac{1}{\theta}\right) \frac{1}{x} f(x \mid \theta) d G(\theta) \\
= & \left(1-\theta_{0}\right) f_{G}(x)-\theta_{0} x \\
& \cdot \int_{\Theta}\left(-\frac{1}{x}\right) f(x \mid \theta) d G(\theta)-\theta_{0} x \\
& \cdot \int_{\Theta} \frac{1}{\theta x} f(x \mid \theta) d G(\theta) .
\end{aligned}
$$

Since $f_{G}(x)=\int_{\Theta}(\theta x)^{-1} \exp \left\{\theta^{-1} \ln x\right\} d G(\theta)$, we get

$$
\begin{aligned}
f_{G}^{(1)}(x)= & \int_{\Theta}\left(-\frac{1}{x}\right) f(x \mid \theta) d G(\theta) \\
& +\frac{1}{\theta x} \int_{\Theta} f(x \mid \theta) d G(\theta) .
\end{aligned}
$$

Thus, we have

$$
\begin{aligned}
\beta(x) & =\left(1-\theta_{0}\right) f_{G}(x)-\theta_{0} x \cdot f_{G}^{(1)}(x) \\
& =u(x) f_{G}(x)+v(x) f_{G}^{(1)}(x),
\end{aligned}
$$

where $f_{G}^{(1)}(x)$ is derivative of $f_{G}(x), u(x)=1-\theta_{0}$, and $v(x)=$ $-\theta_{0} x$.

By (5), Bayes test function is obtained as follows:

$$
\delta_{G}(x)= \begin{cases}1, & \text { if } \beta(x) \leq 0, \\ 0, & \text { if } \beta(x)>0 .\end{cases}
$$

Hence, we can get minimum Bayes risk:

$$
\begin{aligned}
R(G) & =\inf _{\delta} R(\delta, G)=R\left(\delta_{G}, G\right) \\
& =a \int_{\Omega} \beta(x) \delta_{G}(x) d x+C_{G} .
\end{aligned}
$$

If the prior distribution of $G(\theta)$ is known and $\delta(x)=$ $\delta_{G}(x), R(G)$ is achieved. While $G(\theta)$ is unknown, we cannot make use of $\delta_{G}(x)$ and need to introduce EB method.

\section{Construction of EB Test Based on Ranked Set Sampling}

A balanced RSS procedure can be described by Chen et al. [2] . Under the following conditions, we need to construct EB test function. Let $X_{(1) 1}, X_{(1) 2}, \ldots, X_{(1) m}, X_{(2) 1}, X_{(2) 2}, \ldots, X_{(2) m}$, $\ldots, X_{(k) 1}, X_{(k) 2}, \ldots, X_{(k) m}$ be a balanced ranked set sample from population which has the common marginal density function $f_{G}(x)$. We assume perfect ranking. Denote by $X_{(1) 1}, X_{(1) 2}, \ldots, X_{(1) m}, X_{(2) 1}, X_{(2) 2}, \ldots, X_{(2) m}, \ldots, X_{(k) 1}, X_{(k) 2}$, $\ldots, X_{(k) m}$ the historical samples, and $X$ is present sample. Assume $f_{G}(x) \in C_{s, \alpha}, x \in R^{1}$, where $C_{s, \alpha}=\{g(x) \mid g(x)$ is a probability density function and has continuous sth order derivative $g^{(s)}(x)$ with $\left.\left|g^{(s)}(x)\right| \leq \alpha, s \geq 3, \alpha>0\right\}$. First construct estimator of $\beta(x)$.

Let $K_{r}(x)$ be a Borel measurable bounded function vanishing off $(0,1)$ such that

$$
\text { (C1) } \begin{aligned}
& : \frac{1}{t !} \int_{0}^{1} y^{t} K_{r}(y) d y \\
& = \begin{cases}(-1)^{t}, & \text { when } t=r, \\
0, & \text { when } t \neq r, t=0,1,2, \ldots, s-1 .\end{cases}
\end{aligned}
$$

Kernel estimator of $f_{G}(x)$ is defined by

$$
f_{n}^{(r)}(x)=\frac{1}{m k h_{n}^{(1+r)}} \sum_{i=1}^{k} \sum_{j=1}^{m} K_{r}\left(\frac{x-X_{(i) j}}{h_{n}}\right),
$$

where $h_{n}$ is a positive and smoothing bandwidth and $\lim _{n \rightarrow \infty} h_{n}=0$.

Denote by $f_{n}^{(0)}(x)=f_{n}(x), f_{n}^{(r)}(x)$ the $r$ th order derivative of $f_{n}(x)$, for $r=0,1$.

The estimator of $\beta(x)$ is obtained by

$$
\beta_{n}(x)=u(x) f_{n}(x)+v(x) f_{n}^{(1)}(x) .
$$

Hence, EB test function is defined by

$$
\delta_{n}(x)= \begin{cases}1, & \beta_{n}(x) \leq 0, \\ 0, & \beta_{n}(x)>0 .\end{cases}
$$


Let $E$ stand for mathematical expectation with respect to the joint distribution of $X_{(1) 1}, X_{(1) 2}, \ldots, X_{(1) m}, X_{(2) 1}, X_{(2) 2}$, $\ldots, X_{(2) m}, \ldots, X_{(k) 1}, X_{(k) 2}, \ldots, X_{(k) m}$. Hence, we get the overall Bayes risk of $\delta_{n}(x)$ :

$$
R\left(\delta_{n}(x), G\right)=a \int_{\Omega} \beta(x) E\left[\delta_{n}(x)\right] d x+C_{G} .
$$

If $\lim _{n \rightarrow \infty} R\left(\delta_{n}, G\right)=R\left(\delta_{G}, G\right),\left\{\delta_{n}(x)\right\}$ is called asymptotical optimality of EB test function. If $R\left(\delta_{n}, G\right)-R\left(\delta_{G}, G\right)=$ $O\left(n^{-q}\right)$, where $q>0, O\left(n^{-q}\right)$ is asymptotically optimal convergence rate of EB test function $\left\{\delta_{n}(x)\right\}$. Before proving the theorems, we need the following lemmas.

Let $c, c_{1}, c_{2}, c_{3}$ be different constants in different cases even in the same expression.

Lemma 1. Let $X_{(1) 1}, X_{(1) 2}, \ldots, X_{(1) m}, X_{(2) 1}, X_{(2) 2}, \ldots, X_{(2) m}$, $\ldots, X_{(k) 1}, X_{(k) 2}, \ldots, X_{(k) m}$ be balanced ranked set samples. Suppose that (C1) holds, $\forall x \in \Omega$.

(I) When $f_{G}^{(r)}(x)$ is continuous function, $\lim _{n \rightarrow \infty} h_{n}=0$, and $\lim _{n \rightarrow \infty} n h_{n}^{2 r+1}=\infty$, one has

$$
\lim _{n \rightarrow \infty} E\left|f_{n}^{(r)}(x)-f_{G}^{(r)}(x)\right|^{2}=0 .
$$

(II) When $f_{G}(x) \in C_{s, a}$, putting $h_{n}=n^{-1 /(2+s)}$, for $0<\lambda \leq$ 1 , one has

$$
E\left|f_{n}^{(r)}(x)-f_{G}^{(r)}(x)\right|^{2 \lambda} \leq c \cdot n^{-\lambda(s-2 r+1) /(2+s)} .
$$

Proof. Consider the following.

Proof of (I). Using $C_{r}$ inequation, we obtain

$$
\begin{aligned}
& E\left|f_{n}^{(r)}(x)-f_{G}^{(r)}(x)\right|^{2} \\
& \quad \leq 2\left|E f_{n}^{(r)}(x)-f_{G}^{(r)}(x)\right|^{2}+2 \operatorname{Var}\left(f_{n}^{(r)}(x)\right) \\
& \quad:=2\left(A_{1}^{2}+A_{2}\right),
\end{aligned}
$$

where

$$
\begin{aligned}
E f_{n}^{(r)}(x) & =\sum_{i=1}^{k} \sum_{j=1}^{m} n^{-1} h_{n}^{-(r+1)} E\left[K_{r}\left(\frac{x-X_{(i) j}}{h_{n}}\right)\right] \\
& =h_{n}^{-(1+r)} E\left[K_{r}\left(\frac{x-X}{h_{n}}\right)\right] \\
& =h_{n}^{-(1+r)} \int_{0}^{\infty} K_{r}\left(\frac{x-y}{h_{n}}\right) f_{G}(y) d y \\
& =h_{n}^{-r} \int_{0}^{1} K_{r}(u) f_{G}\left(x-h_{n} u\right) d u .
\end{aligned}
$$

Using Taylor expansion, we get

$$
\begin{aligned}
f_{G}\left(x-h_{n} u\right)-f_{G}(u) & \\
= & \frac{f_{G}^{\prime}(x)}{1 !}\left(-h_{n} u\right)+\frac{f_{G}^{\prime \prime}(x)}{2 !}\left(-h_{n} u\right)^{2}+\cdots \\
& +\frac{f_{G}^{(s)}\left(x-\xi h_{n} u\right)}{s !}\left(-h_{n} u\right)^{s} .
\end{aligned}
$$

Because $f_{G}^{(r)}(x)$ is continuous in $x$ and condition (C1), it follows that

$$
\begin{aligned}
0 \leq & \lim _{n \rightarrow \infty}\left|E f_{n}^{(r)}(x)-f_{G}^{(r)}(x)\right| \\
& =\lim _{n \rightarrow \infty}\left|\frac{1}{h_{n}^{r}} \int_{0}^{1} K_{r}(u) f_{G}\left(x-h_{n} u\right) d u-f_{G}^{(r)}(x)\right| \\
& \leq \frac{1}{r !} \int_{0}^{1} u^{r}\left|K_{r}(u)\right| \\
& \cdot \lim _{n \rightarrow \infty}\left|f_{G}^{(r)}\left(x-\xi h_{n} u\right)-f_{G}^{(r)}(x)\right| d u=0 ;
\end{aligned}
$$

further, we have

$$
\lim _{n \rightarrow \infty} A_{1}^{2}=\lim _{n \rightarrow \infty}\left|E f_{n}^{(r)}(x)-f_{G}^{(r)}(x)\right|^{2}=0 .
$$

It is easy to see that

$$
\begin{aligned}
A_{2} & =2 \operatorname{Var}\left[f_{n}^{(r)}(x)\right] \\
& =2 \sum_{i=1}^{k} \sum_{j=1}^{m} n^{-2} h_{n}^{-2(r+1)} \operatorname{Var}\left[K_{r}\left(\frac{x-X_{(i) j}}{h_{n}}\right)\right] \\
& \leq 2 n^{-2} h_{n}^{-2(r+1)} \sum_{i=1}^{k} \sum_{j=1}^{m} E\left[K_{r}\left(\frac{x-X_{(i) j}}{h_{n}}\right)\right]^{2} \\
& \leq c \cdot\left(n h_{n}^{2 r+1}\right)^{-1} .
\end{aligned}
$$

When $h_{n} \rightarrow 0$ and $n h_{n}^{2 r+1} \rightarrow \infty$, we get

$$
\lim _{n \rightarrow \infty} A_{2}=\lim _{n \rightarrow \infty} \operatorname{Var}\left(f_{G}^{(r)}(x)\right)=0 .
$$

Substituting (24) and (26) into (20), proof of (I) is finished.

Proof of (II). Similarly to (20), we can show that

$$
\begin{aligned}
& E\left|f_{n}^{(r)}(x)-f_{G}^{(r)}(x)\right|^{2 \lambda} \\
& \quad \leq 2\left[E f_{n}^{(r)}(x)-f_{G}^{(r)}(x)\right]^{2 \lambda}+2\left[\operatorname{Var} f_{n}^{(r)}(x)\right]^{\lambda} \\
& \quad:=2\left(B_{1}^{2 \lambda}+B_{2}^{\lambda}\right) .
\end{aligned}
$$

By Taylor expansion, we obtain

$$
\begin{aligned}
f_{G}\left(x-h_{n} v\right)= & f_{G}(x)+\frac{f_{G}^{\prime}(x)}{1 !}\left(-h_{n} v\right) \\
& +\frac{f_{G}^{\prime \prime}(x)}{2 !}\left(-h_{n} v\right)^{2}+\cdots \\
& +\frac{f_{G}^{(s)}\left(x-\xi h_{n} v\right)}{s !}\left(-h_{n} v\right)^{s},
\end{aligned}
$$

where $0<\xi<1$, due to condition (C1) and $f_{G}(x) \in C_{s, \alpha}$, we have

$$
\begin{aligned}
& \left|E f_{n}^{(r)}(x)-f_{G}^{(r)}(x)\right| \\
& \quad \leq \int_{0}^{1}\left|K_{r}(v)\right| h_{n}^{s-r} v^{s}\left|\frac{f_{G}^{(s)}\left(x-\xi h_{n} v\right)}{s !}\right| d v \leq c \cdot h_{n}^{s-r} .
\end{aligned}
$$


Therefore, taking $h_{n}=n^{-1 /(2+s)}$, we get

$$
B_{1}^{2 \lambda}=\left|E f_{n}^{(r)}(x)-f_{G}^{(r)}(x)\right|^{2 \lambda} \leq c \cdot n^{-2 \lambda(s-r) /(s+2)} .
$$

By (26), choosing $h_{n}=n^{-1 /(2+s)}$, we can get

$$
B_{2}^{\lambda} \leq\left[c_{1}\left(n h_{n}^{2 r+1}\right)^{-1}\right]^{\lambda} \leq c \cdot n^{-\lambda(s-2 r+1) /(2+s)} .
$$

Substituting (30) and (31) into (27), proof of (II) is finished.

Lemma 2 (see $[8]) . R\left(\delta_{G}, G\right)$ and $R\left(\delta_{n}, G\right)$ are defined by (12) and (17); then

$$
\begin{aligned}
0 & \leq R\left(\delta_{n}, G\right)-R\left(\delta_{G}, G\right) \\
& \leq a \int_{\Omega}|\beta(x)| P\left(\left|\beta_{n}(x)-\beta(x)\right| \geq|\beta(x)|\right) d x .
\end{aligned}
$$

\section{Asymptotic Optimality and Convergence Rates Based on Ranked Set Sampling}

Theorem 3. Assume (C1) and the following regularity conditions hold:

(i) $\lim _{n \rightarrow \infty} n h_{n}^{3}=\infty$;

(ii) $\int_{\Theta} \theta^{-2} d G(\theta)<+\infty$;

(iii) $f_{G}^{(1)}(x)$ is continuous function, and one has

$$
\lim _{n \rightarrow \infty} R\left(\delta_{n}, G\right)=R\left(\delta_{G}, G\right) .
$$

Proof of Theorem 3. Applying Lemma 2, we can get

$$
\begin{aligned}
0 & \leq R\left(\delta_{n}, G\right)-R\left(\delta_{G}, G\right) \\
& \leq a \int_{\Omega}|\beta(x)| p\left(\left|\beta_{n}(x)-\beta(x)\right| \geq|\beta(x)|\right) d x .
\end{aligned}
$$

Put $M_{n}(x)=|\beta(x)| p\left(\left|\beta_{n}(x)-\beta(x)\right| \geq|\beta(x)|\right)$. It is easy to see that $M_{n}(x) \leq|\beta(x)|$.

Again using (10) and Fubini theorem, we obtain

$$
\begin{aligned}
& \int_{\Omega}|\beta(x)| d x \\
& =\int_{\Omega} \int_{\Theta}\left[1-\left(\frac{\theta_{0}}{\theta}\right)^{2}\right] f(x \mid \theta) d G(\theta) d x \\
& =1+\theta_{0}^{2} \int_{\Omega} \int_{\Theta} \theta^{-2} f(x \mid \theta) d G(\theta) d x \\
& =1+\theta_{0}^{2} \int_{\Theta} \theta^{-2} d G(\theta)<+\infty .
\end{aligned}
$$

By domain convergence theorem, then

$$
\begin{aligned}
0 & \leq \lim _{n \rightarrow \infty} R\left(\delta_{n}, G\right)-R\left(\delta_{G}, G\right) \\
& \leq \int_{\Omega}\left[\lim _{n \rightarrow \infty} M_{n}(x)\right] d x .
\end{aligned}
$$

If Theorem 3 holds, we only need to prove $\lim _{n \rightarrow \infty} M_{n}(x)=0$ a.s. $x$.

Applying Markov's and Jensen's inequations, we have

$$
\begin{aligned}
M_{n}(x) \leq & E\left|\beta_{n}(x)-\beta(x)\right| \\
\leq & |u(x)| E\left|f_{n}(x)-f_{G}(x)\right| \\
& +|v(x)| E\left|f_{n}^{(1)}(x)-f_{G}^{(1)}(x)\right| \\
\leq & |u(x)|\left[E\left|f_{n}(x)-f_{G}(x)\right|^{2}\right]^{1 / 2} \\
& +|v(x)|\left[E\left|f_{n}^{(1)}(x)-f_{G}^{(1)}(x)\right|^{2}\right]^{1 / 2} .
\end{aligned}
$$
get

Again using Lemma 1(I), for fixed $x \in \Omega$ and $r=0$, we

$$
\begin{aligned}
0 \leq & \lim _{n \rightarrow \infty} M_{n}(x) \\
\leq & |u(x)|\left[\lim _{n \rightarrow \infty} E\left|f_{n}(x)-f_{G}(x)\right|^{2}\right]^{1 / 2} \\
& +|v(x)|\left[\lim _{n \rightarrow \infty} E\left|f_{n}^{(1)}(x)-f_{G}^{(1)}(x)\right|^{2}\right]^{1 / 2}=0 .
\end{aligned}
$$

Substituting (38) into (36), proof of Theorem 3 is finished.

Theorem 4. Assume (C1) and the following regularity conditions hold.

(C2): consider $\int_{\Omega} x^{-m \lambda}|\beta(x)|^{1-\lambda} d x<+\infty$, where $0<\lambda \leq$ $1, m=0,1$. When $h_{n}=n^{-1 /(2+2 s)}$, where $s \geq 2$, one can obtain

$$
R\left(\delta_{n}, G\right)-R\left(\delta_{G}, G\right)=O\left(n^{-\lambda(s-1) / 2(s+1)}\right),
$$

where $s \geq 2$.

Proof of Theorem 4. Applying Lemma 2 and Markov's inequations, we have

$$
\begin{aligned}
0 \leq & R\left(\delta_{n}, G\right)-R\left(\delta_{G}, G\right) \\
\leq & \int_{\Omega}|\beta(x)|^{1-\lambda} E\left|\beta_{n}(x)-\beta(x)\right|^{\lambda} d x \\
\leq & c_{1} \int_{\Omega}|\beta(x)|^{1-\lambda}|u(x)| E\left|f_{n}(x)-f_{G}(x)\right| d x \\
& \quad+c_{2} \int_{\Omega}|\beta(x)|^{1-\lambda}|v(x)| E\left|f_{n}^{(1)}(x)-f_{G}^{(1)}(x)\right| d x \\
= & D_{n}+E_{n} .
\end{aligned}
$$

By Lemma 1(II) and condition (C2), we get

$$
\begin{aligned}
D_{n} & \leq c_{1} n^{-\lambda s /(2 s+2)} \int_{\Omega}|\beta(x)|^{1-\lambda}|u(x)|^{\lambda} d x \\
& \leq c_{3} n^{-\lambda s /(2 s+2)}, \\
E_{n} & \leq c_{2} n^{-\lambda(s-1) /(2 s+2)} \int_{\Omega}|\beta(x)|^{1-\lambda}|v(x)|^{\lambda}(x) d x \\
& \leq c_{4} n^{-\lambda(s-1) /(2 s+2)} .
\end{aligned}
$$


Substituting (41) into (40), we can obtain $R\left(\delta_{n}, G\right)$ $R\left(\delta_{G}, G\right)=O\left(n^{-\lambda(s-1) / 2(s+1)}\right)$. Proof of Theorem 4 is finished.

Remark 5. When $\lambda \rightarrow 1$ and $s \rightarrow \infty, O\left(n^{-\lambda(s-1) / 2(s+1)}\right)$ nears $\mathrm{O}\left(n^{-1 / 2}\right)$.

\section{Conclusions}

In this paper, we propose the empirical Bayes test rule for the parameter of power distribution. Based on ranked set sampling, the asymptotical optimality and convergence rates of EB test function are obtained. Another extension of this work would consider empirical Bayes inference for some other distributions based on ranked set sampling.

\section{Conflict of Interests}

The authors declare that there is no conflict of interests regarding the publication of this paper.

\section{Acknowledgments}

The authors thank the anonymous referees for constructive suggestions that greatly improved the paper. This work was partially supported by the National Natural Science Foundation of China (11271095 and 11461057) and Natural Science Foundation of Guangdong Ocean University (GDOU2014050217). The work was also supported by the Specialized Research Fund for the Doctoral Program of Higher Education (20124410110002) and National Statistics Projects of China (2012LY178).

\section{References}

[1] G. A. McIntyre, "A method for unbiased selective sampling, using ranked sets," Australian Journal of Agricultural Research, vol. 3, no. 4, pp. 385-390, 1952.

[2] Z. H. Chen, Z. D. Bai, and B. K. Sinha, Ranked Set Sampling: Theory and Applications, Springer, 2003.

[3] H. Robbins, "An empirical Bayes approach to statistics," in Proceedings of the 3rd Berkeley Symposium on Mathematical Statistics and Probability, vol. 1, pp. 157-163, University of California Press, Berkeley, Calif, USA, August 1955.

[4] H. Robbins, "The empirical Bayes approach to statistical decision problems," Annals of Mathematical Statistics, vol. 35, pp. 120, 1964.

[5] R. J. Karunamuni, J. Li, and J. Wu, "Robust empirical Bayes tests for continuous distributions," Journal of Statistical Planning and Inference, vol. 140, no. 1, pp. 268-282, 2010.

[6] X. Xu and D. Zhou, "Empirical Bayes predictive densities for high-dimensional normal models," Journal of Multivariate Analysis, vol. 102, no. 10, pp. 1417-1428, 2011.

[7] X. Li, Y. Shi, J. Wei, and J. Chai, "Empirical Bayes estimators of reliability performances using LINEX loss under progressively type-II censored samples," Mathematics and Computers in Simulation, vol. 73, no. 5, pp. 320-326, 2007.

[8] J. Johns and J. van Ryzin, "Convergence rates for empirical Bayes two-action problems. 2. Continuous case," The Annals of Mathematical Statistics, vol. 43, no. 3, pp. 934-947, 1972.
[9] L. Wang and R. S. Singh, "Linear Bayes estimator for the twoparameter exponential family under type II censoring," Computational Statistics \& Data Analysis, vol. 71, pp. 633-642, 2014. 


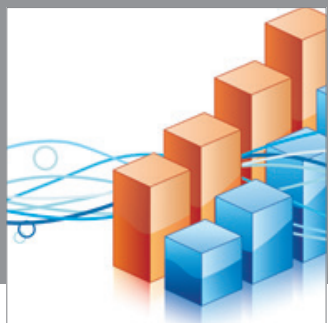

Advances in

Operations Research

mansans

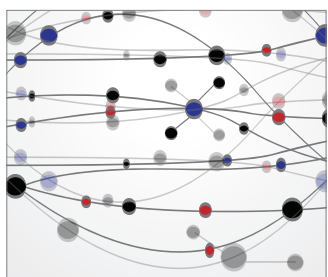

The Scientific World Journal
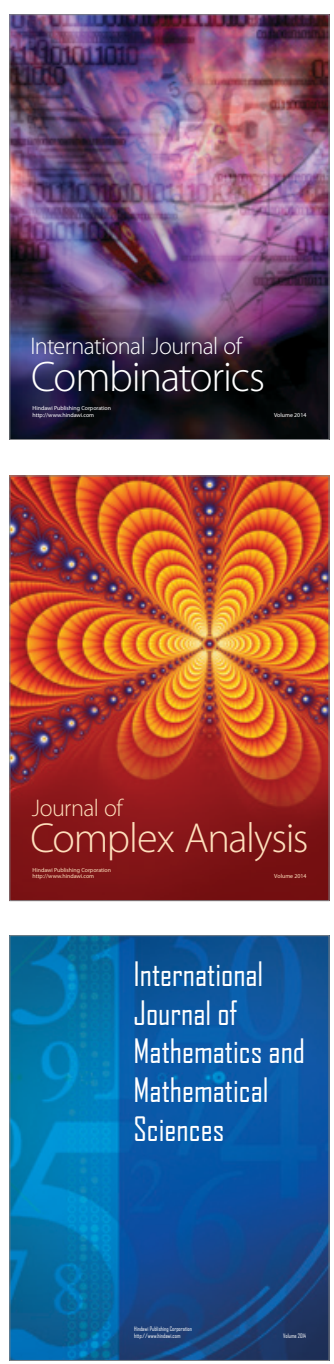
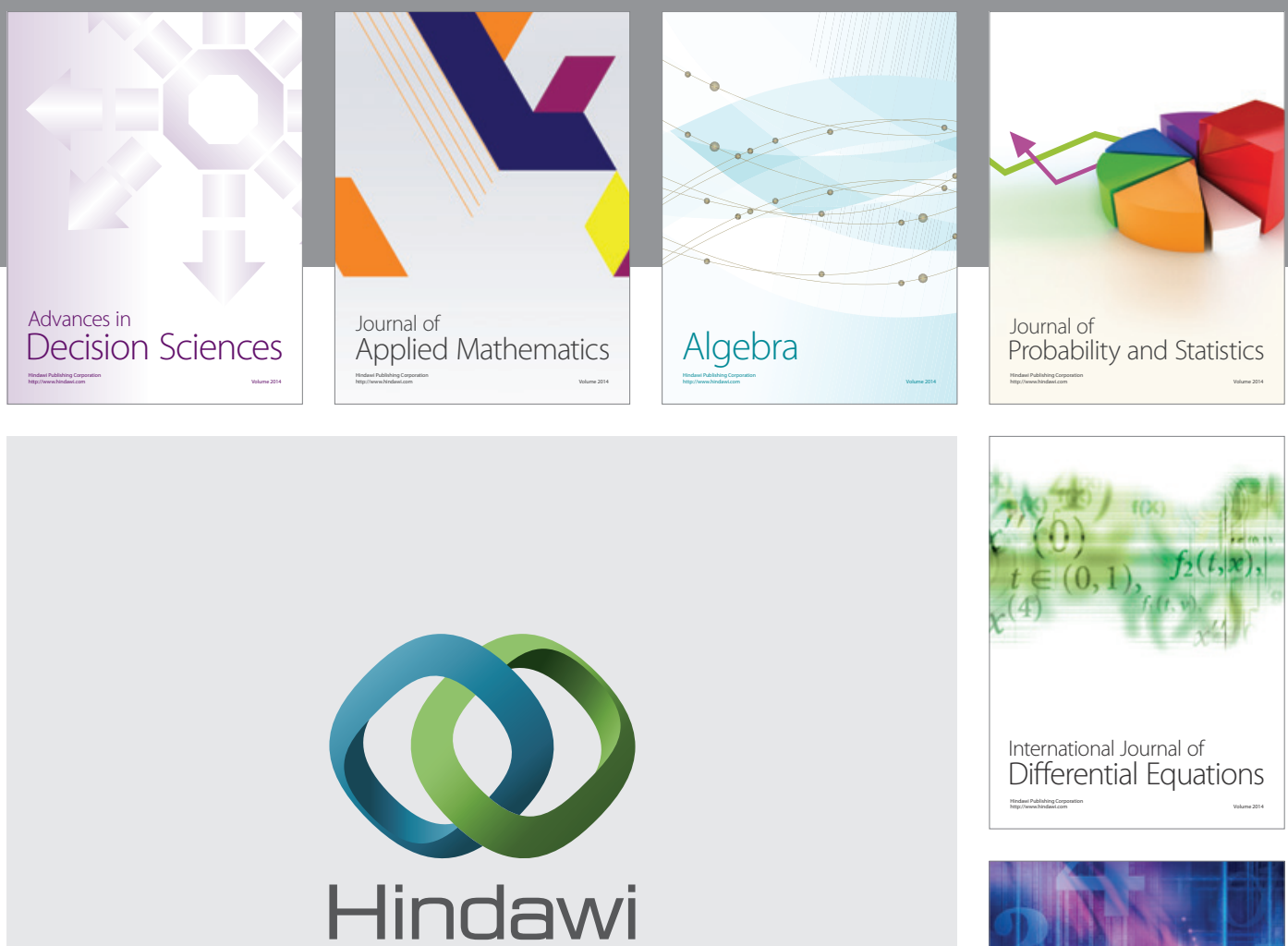

Submit your manuscripts at http://www.hindawi.com
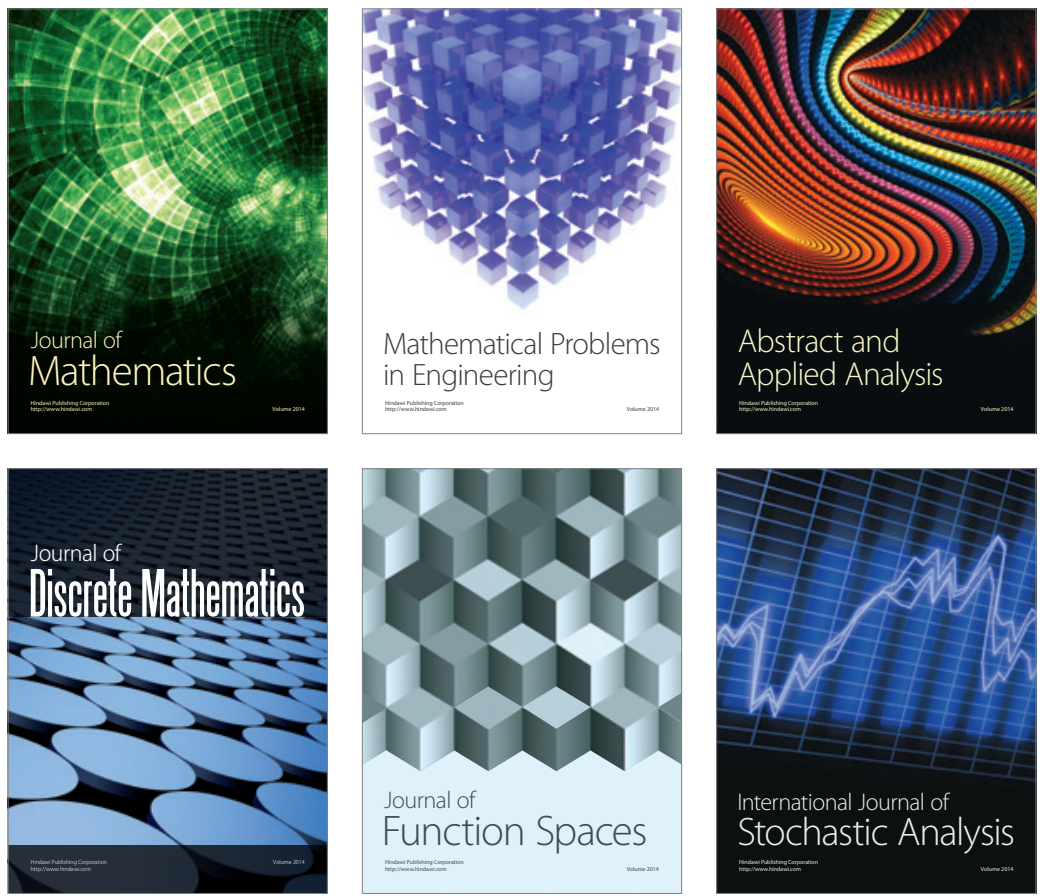

Journal of

Function Spaces

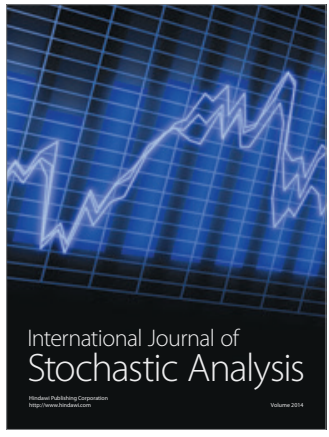

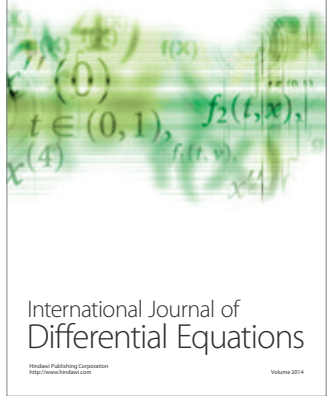
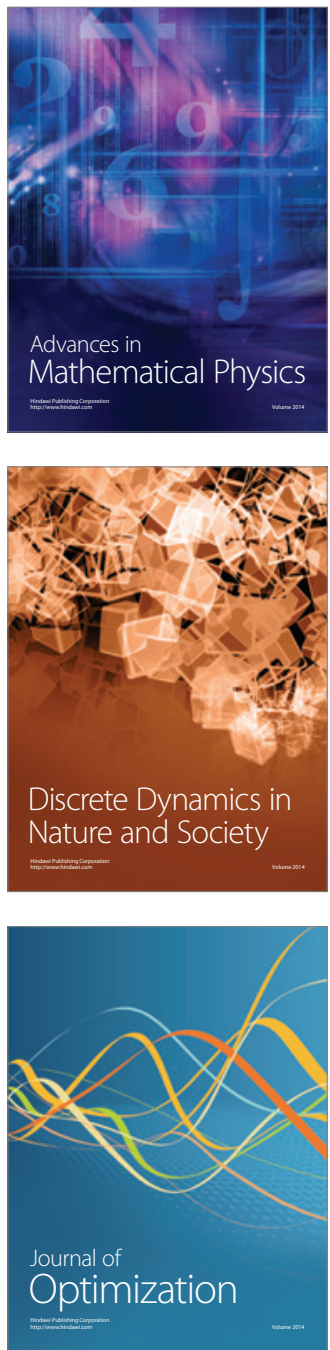